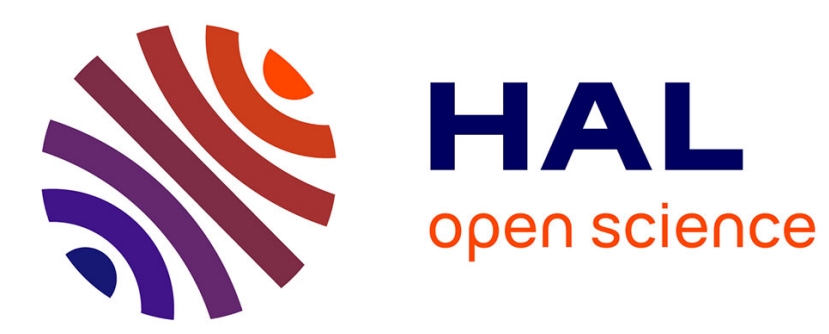

\title{
Thermal conductivity of irradiated porous silicon down to the oxide limit investigated by Raman thermometry and scanning thermal microscopy
}

\author{
Antonin Massoud, P.-O. Chapuis, Bruno Canut, J.-M. Bluet
}

\section{To cite this version:}

Antonin Massoud, P.-O. Chapuis, Bruno Canut, J.-M. Bluet. Thermal conductivity of irradiated porous silicon down to the oxide limit investigated by Raman thermometry and scanning thermal microscopy. Journal of Applied Physics, 2020, 128 (17), pp.175109. 10.1063/5.0020656 . hal-03013854

\author{
HAL Id: hal-03013854 \\ https://hal.science/hal-03013854
}

Submitted on 23 Nov 2020

HAL is a multi-disciplinary open access archive for the deposit and dissemination of scientific research documents, whether they are published or not. The documents may come from teaching and research institutions in France or abroad, or from public or private research centers.
L'archive ouverte pluridisciplinaire HAL, est destinée au dépôt et à la diffusion de documents scientifiques de niveau recherche, publiés ou non, émanant des établissements d'enseignement et de recherche français ou étrangers, des laboratoires publics ou privés. 


\title{
Thermal conductivity of irradiated porous silicon down to the oxide limit investigated by Raman thermometry and scanning thermal microscopy
}

\author{
A.M. Massoud ${ }^{\mathrm{a}, \mathrm{b}}$, P-O. Chapuis ${ }^{\mathrm{b}}$, B. Canut ${ }^{\mathrm{a}}$, J.-M. Bluet ${ }^{\mathrm{a}, \dagger}$ \\ ${ }^{a}$ Univ Lyon, Institut des Nanotechnologies de Lyon (INL), CNRS, INSA de Lyon, F-69621 Villeurbanne, \\ France. \\ b Univ Lyon, CNRS, INSA-Lyon, Université Claude Bernard Lyon 1, CETHIL UMR5008, F-69621, \\ Villeurbanne, France.
}

Irradiating porous silicon is expected to reduce thermal conductivity without altering the porous structure and can be studied by optical techniques provided optical properties can be established reliably. Toward this end, meso-porous silicon (PSi), with a porosity of 56\%, was prepared from $\mathrm{p}^{+} \mathrm{Si}$ wafer $\left(0.01-0.02 \Omega . \mathrm{cm}^{-1}\right.$ resistivity) and was partially amorphized by irradiation in the electronic regime with ${ }^{129} \mathrm{Xe}$ ions at two different energies (29 MeV and $91 \mathrm{MeV}$ ) and five fluences ranging from $10^{12} \mathrm{~cm}^{-2}$ to $3.10^{13} \mathrm{~cm}^{-2}$. The PSi structure is monitored by scanning electron microscopy. High-resolution transmission electron microscopy shows that the amorphous phase is homogeneous in volume and that there is no formation of amorphous-crystalline core-shell structures. An agreement is found between the thermal conductivity results obtained with microRaman thermometry, which is an optical contactless technique heating the sample in the depth, and scanning thermal microscopy, which is an electrical technique heating the sample by contact at the sample surface. A linear relation is established between the effective thermal conductivity and the amorphous fraction, predicting the thermal conductivity of fully-amorphous porous $\mathrm{Si}$ below $1 \mathrm{~W} \cdot \mathrm{m}^{-1} \cdot \mathrm{K}^{-1}$. The obtained values are comparable to that of $\mathrm{SiO}_{2}$, reduced by a factor 6 in comparison to non-irradiated porous samples $\left(\sim 6.5 \mathrm{~W} \cdot \mathrm{m}^{-1} \cdot \mathrm{K}^{-1}\right)$ and smaller than bulk silicon by more than two orders of magnitude.

${ }^{\dagger}$ corresponding author : jean-marie.bluet@insa-lyon.fr

\section{Introduction}

With the development of 3D integration in micro/nanoelectronics, the development of low thermal conductivity materials compatible with CMOS technology has become increasingly important in recent years. Furthermore, the development of energetically autonomous nodes in the Internet Of Things (IOT) has given a renewed interest for CMOS compatible materials exhibiting thermoelectric conversion $^{1,2}$. Thermal insulation is also essential in many types of micro electromechanical systems (MEMS). It can help reducing power consumption, decrease response time, or improve sensitivity. Moreover, insulating hot areas from the rest of the device is crucial in a system-on-chip.
Presently-available thermal insulating material configurations, such as suspended silicon membranes ${ }^{3,4,5}$, are difficult to integrate when thick layers or high mechanical stability are required in Si-based devices.

Porous $\mathrm{Si}(\mathrm{PSi}$ ) nanostructures have already been reported to be used as thermal insulating materials for the manufacturing of thermal sensors or integrated devices and are compatible with CMOS technology $y^{6-10}$. Thick PSi layers with high porosities $(>75 \%$ ), prepared by electrochemical anodization, can present a thermal conductivity ( $\lambda_{P S i}$ ) two orders of magnitude lower than that of bulk crystalline $\mathrm{Si}$ $(\mathrm{c}-\mathrm{Si})^{11,12,13}$. Indeed, the porosity $P$ (void fraction) of PSi combined with the phononphonon scattering due to the formation of nanometric dendrites, lead to low thermal 
conductivity in PSi. Furthermore, percolation with the heat forced to flow across constrictions in the dendritic structures, reduces even more $\lambda_{P S i}$, which was shown to decrease cubically with $P^{14}$. At the same time, the PSi hardness also diminishes with its porosity following a $(1-P)^{2 / 3}$ law ${ }^{15}$ for porosities up to $75 \%$, and even more rapidly, for higher porosities ${ }^{16}$. Young's modulus also decreases with porosity ${ }^{17,18}$. According to the values of ref. 17, measured up to $90 \%$ porosity for a constant PSi thickness corresponding to our samples $(10 \mu \mathrm{m})$, it diminishes rapidly with porosity with a $(1-\mathrm{P})^{2.1}$ law. In the case of ref. 18 no values was measured above $60 \%$ porosity because the samples were too brittle. Nevertheless, operating CMOS compatible devices, using PSi thick layers were demonstrated ${ }^{8-10}$. It's worth noticing that in these devices the PSi is used as a buried layer in a box which may limit the mechanical stress. In ref. 9, PSi with porosity $75 \%$ has been oxidized which may enhance its hardness ${ }^{18}$, while in ref. 10 a cap $\mathrm{SiO}_{2}$ layer was deposited on top of the $150 \mu \mathrm{m} 76 \%$ porosity PSi layer.

Consequently, for MEMS application where the PSi thermal insulating layer may be stressed mechanically, a trade-off between the material thermal conductivity and its mechanical strength has to be found. Toward this end, it was shown, using Raman Spectroscopy, that a greater decrease in the thermal conductivity of PSi can be obtained by amorphizing its crystalline phase using uranium irradiation at $110 \mathrm{MeV}^{19}$. This result was confirmed by Isaiev et al. by photoacoustic technique ${ }^{20}$.

Up to now, in most of the experimental studies of porous silicon only one thermal characterization technique was applied for the determination of $\lambda_{P S i}$. Cruz-Orea et al. and Isaiev et al. measured $\lambda_{P S i}$ by the photoacoustic technique ${ }^{21,20}$. Scanning thermal microscopy (SThM) was used by Gomes et al. for the determination of $\lambda_{P S i}$ of thin layers ${ }^{22}$. Gesele $e t$ al., Valalaki et al., and J.H. Seol et al. used the
$3 \omega$ technique for the same aim ${ }^{14,23,24}$. Using micro-Raman thermometry ( $\mu-\mathrm{RT})$ and SThM, Newby et al. showed a discrepancy between the two techniques in the thermal conductivity of irradiated PSi (IPSi) samples with $P=41 \%^{19}$. In this first study PSi samples were irradiated in the electronic regime (i.e. using projectile with an energy higher than $10 \mathrm{keV}$ per nucleon) at one energy (110 MeV) using uranium ions at six different fluences.

In this work we propose to study further, by Raman Spectrosocy and SThM results the evolution of the thermal conductivity of PSi irradiated by swift heavy ions in the electronic regime. The aim of using both technique is twofold:

(i) As Raman is more sensitive to volume and SThM to surface we expect to correlate the homogeneity of the samples morphology measured by TEM with their thermal properties ;

(ii) As each of the technique may be prone to uncertainty and systematic errors, the comparison of the results is expected to provide reliable and reproducible.

Samples were irradiated at different energies and fluences. In comparison to ref. 19 lighter ${ }^{129} \mathrm{Xe}$ ions were used at two different energies (91 and $29 \mathrm{MeV}$ ) in order to investigate the impact of ion weight and energy on irradiated sample thermal conductivity. The energy per nucleon of $225 \mathrm{keV}$ for $29 \mathrm{MeV}{ }^{129} \mathrm{Xe}$ is more than two times smaller than the one used in ref. 19. We find that the SThM and the $\mu$-RT results agree very well. By this way we obtain precise evolution of $\lambda_{\mathrm{PSi}}$ with amorphization rate and we can discuss in particular the obtained values for crystalline PSi and fully amorphized PSi. It is shown that thermal conductivity values can reach the oxide limit.

\section{Experimental details}

\section{A. Manufacturing process of the samples}


PSi layers were formed by electrochemical etching of $500 \mu \mathrm{m}$ thick $<100>$ monocrystalline boron-doped p+ silicon wafers with resistivity in the range $0.01-0.02 \Omega . \mathrm{cm}^{-1}$ (this parameter was fixed and is not subject to a particular investigation). The choice of a $p+$ type wafer was based on the goal of obtaining mesoporous silicon, with crystallites diameter average size lower than $20 \mathrm{~nm}$. The electrolyte consists of $48 \mathrm{wt} \% \mathrm{HF}$ and pure ethanol in a volume ratio of $1: 1$. The average crystallite diameter was found to be $11 \mathrm{~nm}$, based on the full width at half maximum of the Stokes Raman peak of the non-irradiated PSi (non-IPSi) sample ${ }^{25}$. This kind of porous silicon is known to have, in general, a low thermal conductivity with good mechanical properties, making it a good candidate for MEMS applications. The obtained PSi layer was $10 \mu \mathrm{m}$ thick, with $\mathrm{P}=56 \%$ deduced from reflectivity measurements and the application of the Looyenga-LandauLifshitz (LLL) effective medium model in the porous layer.

Irradiation of the ten samples was carried out at the IRRSUD beamline of the GANIL accelerator by ${ }^{129} \mathrm{Xe}$ at two different energies, $E_{1}=91 \mathrm{MeV}$ and $E_{2}=29 \mathrm{MeV}$, and at five different ion fluences $10^{12}, 3 \times$ $10^{12}, 6 \times 10^{12}, 10^{13}$ and $3 \times 10^{13} \mathrm{~cm}^{-2}$.

\section{B. Amorphous phase distribution}

In order to investigate the interaction mechanisms of irradiating ions with the porous structure, scanning electron microscopy (SEM) and high-resolution transmission electron microscopy (HRTEM) experiments were carried out. The amorphous phase fraction was determined using micro-Raman spectroscopy $^{26}$. The method consists in reproducing the Raman spectrum with one component for the amorphous phase corresponding to a peak centered at $480 \mathrm{~cm}^{-}$
1 and a multi-component fit (4 Gaussian curves) for the crystalline PSi peak, characterized by a peak asymmetrically broadened and slightly shifted towards low energies in comparison to bulk Si (see FIG. 1). Considering the Raman oscillator strength of PSi and amorphous $\mathrm{Si}$ to be equal, the amorphous fraction is then deduced from the integrated intensity ratio of the $480 \mathrm{~cm}^{-1}$ peak to the crystalline PSi one. SEM images of, respectively non-IPSi, IPSi at the lowest fluence $10^{12} \mathrm{~cm}^{-2}$ for both energies and, at the highest fluence $3 \times 10^{13}$ $\mathrm{cm}^{-2}$ still for both energies are given in FIG. 2 (a,b), (c,d) and (e,f). FIG. 2 (c,d,e,f)) were taken at the same magnification focusing the electron beam in the middle of the sample. FIG. 2a, at low magnification, shows the sample structure with a well-defined PSi layer. In addition to the presented ones, three different images were taken for each sample: close to the surface, in the middle and at the bottom of the porous layer (not shown here). A retention of the sponge-like structure of PSi (FIG. 2.b) is observed in FiG. 2. (c, d, e and $\mathrm{f}$ ). The same was found for the 10 irradiated samples with no difference from the top of the PSi layer down to the bottom near the $\mathrm{p}^{+} \mathrm{Si}$ substrate. In particular no cracks or porous structure destruction, due to nuclear interaction of the Xe projectiles, was observed in the depth of the PSi layer, confirming that the interaction, within the major part of the projectile path, is mainly governed by electronic processes for both energy and the five fluences. This indicates that the reduction in thermal conductivity is not due to sample cracks or structural destruction. While the sponge-like structure of PSi is maintained, a change in the dendrites shape is observed. Indeed, their extremity turned into beads for the highest irradiation fluence of $3 \times 10^{13} \mathrm{~cm}^{-2}$ for both irradiation energies (see FIG. 2 (e,f)).

This can be explained by the ion interaction with the dendrite extremity in the electronic 
regime $^{27}$. Indeed, after ion interaction with the electrons of the target, the deposited energy converts into heat, which can induce latent track formation along the projectile paths ${ }^{28,29}$. For intense electronic excitations and a strong electron-phonon coupling, a cylindrical zone is created along the ions path. Typically after 100 ps, this region is going to cool back down and gets back to room temperature with an amorphous phase that differs from the initial one. The amorphous path is called ion track ${ }^{30,31}$. This phenomenon is enhanced in porous material due to thermal spike effect in the dendrite's extremity. In order to observe if the whole dendrite is homogeneously amorphized, or if the core remains crystalline, cross-sectional HRTEM was performed on non-irradiated (FIG. $2(\mathrm{~g}))$ and irradiated PSi at the highest energy and fluence (see FIG. 2 (h)) on lamellae prepared by means of focused ion beam. Both images were taken in the middle of the samples. The presence of a periodic crystal in FIG. 2 (g) is indicated by the vertical lines, whereas it is clearly visible that the vertical lines have almost disappeared in FIG. 2 (h) as an indication of the amorphization of the sample (here $83 \%$ ). In order to determine if the volume of the irradiated sample is homogenously amorphous without a spatial distribution like a core-shell structure in the dendrites, HRTEM images were carried out on the top, middle, and bottom of the irradiated layer, and along $50 \mathrm{~nm}$ in the direction perpendicular to the dendrites (images not shown here). We observed that, for all these experiments, the amorphous phase is present.

\section{Two techniques to determine the thermal conductivity}

\section{Micro-Raman Thermometry}

$\mu$-RT is based on micro-Raman spectroscopy which is an optical and nondestructive technique (see FIG. 3 (a) and the inset of FIG. 4). We used a Renishaw Raman microscope equipped with a doubled frequency continuous YAG laser beam with a wavelength of $532 \mathrm{~nm}$ and an objective $\times 50$ with a numerical aperture of 0.5 . The measured laser spot diameter is $2 \sigma=3.8 \mu \mathrm{m}$ (using the knife-edge technique ${ }^{27}$ ). $\mu$-RT consists in measuring the sample temperature by monitoring the Stokes line position of the material for different laser incident power.

This requires on the one hand to determine quantitatively the power absorbed by the sample and its spatial distribution and, on the other hand, to precisely determine the temperature rise form the Raman peak shift. The laser power was measured using a photometer placed at the sample position and the absorption of $\mathrm{P}=56 \%$ PSi was measured to be 0.9 using an integrating sphere. The Si Raman line shift was converted to temperature using the linear dependence established ${ }^{33}$ for low temperature rise $(<100 \mathrm{~K})$ in the case of crystalline $\mathrm{Si}$ :

$$
\Delta T=0.022 \times \Delta \omega,
$$

where $\Delta T$ is the temperature rise in kelvins and $\Delta \omega$ is Stoke line shift in $\mathrm{cm}^{-1}$. We assumed that this value of 0.022 was adequate for the crystalline part of our PSi irradiated samples as it was shown to be the same for $\mathrm{PSi}^{34}$ partially oxidized $\mathrm{PSi}^{35}$ and $\mathrm{Si}$ nanowires $^{36}$. The results on the nonirradiated sample and for an amorphized one with the high fluence of $3 \times 10^{13} \mathrm{~cm}^{-2}$ at $E_{1}$ energy are provided in FIG. 4 . The slopes of the curves (obtained from linear fit) in FIG. 4 represent the thermal resistances. The one for the irradiated (amorphized) sample is obviously higher. The last step for thermal conductivity $(\lambda)$ determination (from the measured thermal resistance) is to calculate the thermal resistance as a function of $\lambda$, which depends for each laser power on the isothermal contour and the temperature rise under the laser spot. Toward this end, 
in order to take into account the limited PSi thickness $(10 \mu \mathrm{m})$ in comparison to the laser penetration depth $(1.2 \mu \mathrm{m})^{37}$ and also the Gaussian profile of the laser spot, we have used finite element method for solving the heat equation by including volume heat source:

$$
P(r, z)=\frac{2 A P_{0}}{\pi \sigma^{2}} \alpha_{a} \mathrm{e}^{-\frac{2 r^{2}}{\sigma^{2}}} \mathrm{e}^{-\mathrm{z} / \alpha_{\mathrm{a}}}
$$

where $A$ is the absorbance (0.9), $\sigma$ is the laser beam radius at $1 / \mathrm{e}^{2}$ of the total power intensity $(1.9 \mu \mathrm{m}), \alpha$ is the absorption coefficient for PSi with $\mathrm{P}=56 \%\left(8300 \mathrm{~cm}^{-1}\right.$ $\left.{ }^{37}\right) . P_{O}$ is the incident laser power. This way we determined the real shape of the thermal profile with a $2 \mathrm{D}$ axial symmetry around the laser beam center (see FIG. 3 (b)). Due to the lateral profile of the beam, the thermal resistance deviates from the usual $1 /(4 \lambda R)$ value obtained for isothermal sources, where $R$ is the source radius. The depth-dependent absorption induces also a deviation in comparison to a full-analytical solution involving surface absorption with a Gaussian beam.

\section{Scanning thermal microscopy}

The second thermal probing technique used is SThM. The setup considered is an atomic force microscopy (AFM) configuration, where a thermoresistive tip is mounted on a cantilever. In this study, a thermoresistive Wollaston probe was used. The probe is connected to an electrical circuit in a Wheatstone bridge configuration in order to precisely determine its resistance. The probe is operated in the so-called active mode using a DC current, which is the most widely used method ${ }^{38}$. The Wollaston wire is set far from the sample and heated up by the Joule effect. The Wheatstone bridge is then balanced and the electrical probe power far from contact, $P_{f f c}$ is determined (see FIG. 5 (a)). Next, the tip is brought into contact with the sample which is at room temperature. The heat flows from the tip to the sample causing a decrease in the tip temperature and so a change in the tip electrical resistance. This decrease in temperature is directly related to the thermal conductivity of the studied sample and induces a change in the output voltage of the Wheatstone bridge. The Wheatstone bridge is then balanced and the electrical probe power in contact, $P_{i c}$ is determined (see FIG. 5 (b)). The resulting dissipated probe power, between far-from-contact and contact tip positions, is then calculated. By plotting the relative dissipated probe power $\frac{\Delta P}{P}=\frac{P_{i c}-P_{f f c}}{P_{i c}}$ as a function of the thermal conductivity of calibration samples $^{38,39}, \lambda_{P S i}$ and $\lambda_{I P S i}$ were obtained (see FIG. 5(c) for the procedure). We note that we did not account for the roughness that might arise on the top surfaces of the porous silicon samples, as the used calibration samples were polished and have flat surfaces. This is possible because the heat flux depends hardly on the contact with this probe, as most of it flows through the air around the probe ${ }^{32}$. As a result, the water meniscus effect at the tip sample interface is expected to be small (in contrast to vacuum measurements, where it plays a more important role). Indeed, in Fig. 5, where hydrophilic and hydrophobic materials are considered, no clear effect of the meniscus can be observed. Another remark is linked to the shape of the curve in FIG. 5(c), which does not level off for thermal conductivities larger than $3 \mathrm{~W} . \mathrm{m}^{-}$ ${ }^{1} \cdot \mathrm{K}^{-1}$ and allows for measurements at least up to $6 \mathrm{~W} \cdot \mathrm{m}^{-1} \cdot \mathrm{K}^{-1}$. This is investigated in another work ${ }^{40}$. 


\section{Impact of irradiation on thermal conductivity}

The two techniques were carried out on the ten irradiated samples and on the nonirradiated one in an open environment at room temperature. The results are displayed in FIG. 6.

\section{A. Experimental results}

The histogram in FIG. 6 shows the evolution of the effective thermal conductivity of PSi while increasing the irradiation fluence for the two irradiation energy. Error bars for SThM were plotted considering the maximum and the minimum in the dissipated probe power while for $\mu$-RT considering $\pm 10 \%$ of each obtained value. The results obtained from $\mu-\mathrm{RT}$ and SThM measurements are in very good agreement. The small differences, which are within the error bars, between the results obtained from the two techniques could origin from the exact thermal conductivity of the layer which was not determined directly for SThM: a small portion of the signal could be due to the substrate. Another possibility is the weak impact of the sample roughness, which is not taken into account in SThM. The two techniques show a decrease in $\lambda_{\text {IPSi }}$ while increasing the irradiation fluence. At a given fluence, a lower (approximately 10\%) $\lambda$ is achieved for the highest irradiation energy. Furthermore, a linear relation is established between our IPSi samples and the amorphous fraction (see FIG. 7). The lowest $\lambda_{\text {IPSi }}=1.2$ $\mathrm{W} \cdot \mathrm{m}^{-1} \cdot \mathrm{K}^{-1}$ obtained in this study is more than 5.3 times smaller than that of the non-IPSi one $\left(6.4 \mathrm{~W} \cdot \mathrm{m}^{-1} \cdot \mathrm{K}^{-1}\right)$ and more than two orders of magnitude smaller than that of bulk $\mathrm{Si}$. It is comparable to that of $\mathrm{SiO}_{2}, \lambda_{\mathbf{S i O}_{2}}=1.2 \mathrm{~W} \cdot \mathrm{m}^{-}$ ${ }^{1} \cdot \mathrm{K}^{-1} 41,42,43,44$, and is close to $1.1 \mathrm{~W} \cdot \mathrm{m}^{-1} \cdot \mathrm{K}^{-1}$, the effective thermal conductivity of $\mathrm{PSi}$ with $\mathrm{P}=75 \%{ }^{19}$. This means that by irradiating PSi of medium porosity (good mechanical stability) we can obtain thermal conductivities values close to the ones of non-IPSi of higher porosity, thus avoiding mechanical strength problems.

\section{B. Comparison with models and discussion}

The effective thermal conductivities of our samples can be fitted linearly as a function of the amorphous phase for the two irradiation energies and for the two characterization techniques used in this work (see FIG. 7).

The same slopes are obtained with an intercept with a relative variation of $10 \%$. This variation is related to the very small difference in the nonirradiated PSi effective thermal conductivities obtained by each technique. The obtained equation is

$$
\lambda_{I P S i}=\gamma(1-a)+\beta
$$

where $a$ is the amorphous fraction in the matter, $\gamma=5.67 \mathrm{~W} \cdot \mathrm{m}^{-1} \cdot \mathrm{K}^{-1}$ and $\beta=\lambda_{I P S i 100 \%}$. is the thermal conductivity of the irradiated PSi with $a=100 \%$. The negative slope is an indication of the reduction of $\lambda_{I P S i}$ when increasing the irradiation fluence. From $\mu$ $\operatorname{RT}\left(\beta_{R}\right)$ and $\operatorname{SThM}\left(\beta_{S}\right)$ linear fits, we find $\beta \in[0.7-1] \mathrm{W} \cdot \mathrm{m}^{-1} \cdot \mathrm{K}^{-1}$. Making $\alpha=0$ in Eq. (3) we obtain the thermal conductivity for non-irradiated PSi at $56 \%$ which is found to be $6.3 \pm 0.2 \mathrm{~W} \cdot \mathrm{m}^{-1} \cdot \mathrm{K}^{-1}$. It is difficult to find in literature the same PSi sample as the one we manufactured, but this non-IPSi thermal conductivity is in good agreement with already published results ${ }^{14,19,20,22,24}$.

We now compare our results with the model that allows obtaining $\lambda_{\text {IPSi }}$ as a function of the amorphous fraction developed by Newby et $a l .{ }^{14}$. In this model, the partially-amorphous porous silicon structure is considered as parallel wires of amorphous and crystalline phases. While this analysis can be questioned, it is still informative. The effective thermal conductivity of a partially amorphous PSi layer (Eq. (3)) can be rewritten as ${ }^{19}$ : 


$$
\begin{gathered}
\lambda_{I P S i}=(1-P)\left[\lambda_{S i}(1-a)\right. \\
\left.+\lambda_{a S i} a\right],
\end{gathered}
$$

where $\lambda_{s i}, \lambda_{a s i}$ are the effective thermal conductivities of, respectively, the crystalline $\mathrm{Si}$ nanowires and the amorphous $\mathrm{Si}$ ones. Theoretical studies demonstrated that amorphous Si thermal conductivity can vary between 1 and $5 \mathrm{~W} \cdot \mathrm{m}^{-1} \cdot \mathrm{K}^{-1}{ }^{45}$. To our best knowledge, no experimental value for $\lambda_{S i}$, at room temperature, was obtained for nanowires of $11 \mathrm{~nm}$ in diameter (the diameter of our $\mathrm{Si}$ crystallites). We applied this model to fit our experimental data obtained by SThM for $E_{2}$, while keeping $\lambda_{S i}$ as a variable and $\lambda_{a s i} \epsilon[1 ; 5]$ $\mathrm{W} \cdot \mathrm{m}^{-1} \cdot \mathrm{K}^{-1}$. The resulting best fit gives $\lambda_{S i}=$ $15.2 \mathrm{~W} . \mathrm{m}^{-1} \cdot \mathrm{K}^{-1}$ and $\lambda_{a s i}=2.3 \mathrm{~W} . \mathrm{m}^{-1} \cdot \mathrm{K}^{-1}$ with $R^{2}=0.98$. With this model, the crystallites of $11 \mathrm{~nm}$ in diameter turned out to have $\lambda_{S i}$ larger than that of single-crystalline silicon nanowires of $22 \mathrm{~nm}$ in diameter $\left(\sim 10 \mathrm{~W} \cdot \mathrm{m}^{-1} \cdot \mathrm{K}^{-1}{ }^{46}\right)$. Other modified effective medium approaches (EMA) such as Maxwell-Garnett, Bruggeman and LLL, where the mean free paths in the crystalline phase can be considered further reduced due to confinement ${ }^{47,48}$, also predict low thermal conductivities, whatever the geometry of the crystallites (which can be asymptotically considered as spheres or cylinders). The impact of optical modes in confined structures might be significant ${ }^{49}$.The difficulty in matching the models derived from the Boltzmann transport equation with up-to-date mean free path distributions and the experimental results for crystalline porous samples has already been highlighted ${ }^{24}$.

\section{Conclusions and prospects}

Using HRTEM we have showed that a homogenous amorphization in volume takes place when irradiating PSi samples with swift heavy ions. We have demonstrated a very good agreement between $\mu$-RT (optical, contactless technique with a volume heat source) and SThM (electrical and contact technique) measurements performed on PSi and IPSi at two different energies. This agreement shows that both technique are not affected by systematic errors. For the Raman part it validates the effective medium approach for the calculation of absorption in irradiated PSi together with the Raman shift variation with temperature (considered as the same than for bulk $\mathrm{Si}$ ). For the SThM it shows that the rough mechanical contact does not play a significant role for thermal conductivity materials probed in air by micro-probes.

We have showed a decrease in irradiated PSi thermal conductivity by a factor of more than 5.3 and a decrease by more than two orders of magnitude in comparison with PSi and bulk $\mathrm{Si}$ respectively. The lowest effective thermal conductivity that was obtained is $1.2 \mathrm{~W} \cdot \mathrm{m}^{-1} \cdot \mathrm{K}^{-1}$ close to that of highly porous silicon $(\mathrm{P}=75 \%)$, avoiding mechanical fragility due to high porosity in our case. Indeed, according to ref. 17, the Young modulus for a $56 \%$ porosity, $10 \mu \mathrm{m}$ thick PSi layer is $24 \mathrm{GPa}$ while the one for $75 \%$ porosity is reduced to $7 \mathrm{GPa}$. Nevertheless, the Young modulus of amorphous $\mathrm{Si}$ maybe lower than that of crystalline $\mathrm{Si}$ (a factor 2 can be considered ${ }^{50}$ despite scattered values in the literature). This value of $1.2 \mathrm{~W} \cdot \mathrm{m}^{-1} \cdot \mathrm{K}^{-1}$ is also comparable with thermal conductivity of $\mathrm{SiO}_{2}$, one of the usual solid insulators.

We have found a linear relation between the effective thermal conductivity and the amorphous fraction. This may hold the key to thermal conductivity prediction if the amorphous fraction is known. However, the match with effective media approaches and spectral distribution of mean free paths of the bulk is difficult, calling for improved modelling steps.

The predicted thermal conductivity value for a fully-amorphized sample ranges between $0.7 \mathrm{~W} \cdot \mathrm{m}^{-1} \cdot \mathrm{K}^{-1}$ and $1 \mathrm{~W} \cdot \mathrm{m}^{-1} \cdot \mathrm{K}^{-1}$, which is 
between $16 \%$ and $40 \%$ lower than the one for non-IPSi with $\mathrm{P}=75 \%$, while the Young's modulus, is approximately twice, according to previously given literature results. In order to confirm this potential interest of irradiated PSi for MEMS application, a study of the mechanical properties will be useful, in particular to investigate the effect of amorphization on hardness and Young's modulus.

\section{Acknowledgements}

The authors acknowledge support from INSA Lyon through BQR project MaNaTherm. Support was also obtained from programme NANO2017. We thank Dr. S. Gomes, Dr. B. Sadani and Dr. P. Newby for useful discussions. We thank also Dr. L. Roiban for his help in HRTEM experiments and D. Troadec for TEM lamellae preparation.

\section{Data availability Statement}

Data available on request from the authors

\section{References}

1 J. Tang, H. T. Wang, D. H. Lee, M. Fardy, Z. Huo, T. P. Russell, and P. Yang, Nano Lett., vol. 10, no. 10, pp. 4279-4283 (2010).

2 V. Lacatena, M. Haras, J. F. Robillard, S. Monfray, T. Skotnicki, and E. Dubois, Microelectron. Eng., vol. 121, pp. 131134 (2014).

3 M. Nomura, J. Nakagawa, Y. Kage, J. Maire, D. Moser and O. Paul, Appl. Phys. Lett., 106, 14 (2015).

4 P. E. Hopkins, C. M. Reinke, M. F. Su, R. H. Olsson, E. A. Shaner, Z. C. Leseman, J. R. Serrano, L. M. Phinney, and I. El-Kady, Nano Lett., vol. 11, no. 1, pp. 107-112 (2011).
5 E. Chavez- Ángel, J. S. Reparaz, J. GomisBresco, M. R. Wagner, J. Cuffe, B. Graczykowski, A. Shchepetov, H. Jiang, M. Prunnila, J. Ahopelto, F. Alzina, and C. M. Sotomayor Torres, et al., APL Mater., vol. 2, no. 1 (2014).

6 V. Lysenko, S. Perichon, B. Remaki, and D. Barbier, Sens. Actuators, A 99, 13 (2002).

7 G. Kaltsas and A. G. Nassiopoulou, Sens. Actuators, A 76, 133 (1999).

8 G.Kastlas, A. Nassiopoulos and A. Nassiopoulou, IEEE Sensors Journal 2(5), 463-475(2002).

9 E. Hourdakis and A. Nassiopoulou, Sensors 13, 13596-13608 (2013)

10 P. Sarafis and A. Nassiopoulou, Nanoscale Research Letters, 9:418 (2014)

$11 \mathrm{G}$. Benedetto, L. Boarino, and R. Spagnolo, Appl. Phys. A Mater. Sci. Process., vol. 64, no. 2, pp. 155-159 (1997).

12 V. Lysenko, S. Périchon, B. Remaki, D. Barbier, and B. Champagnon, J. Appl. Phys., vol. 86, no. 12, p. 6841 (1999).

13 S. Périchon, V. Lysenko, P. Roussel, B. Remaki, B. Champagnon, D. Barbier, and P. Pinard, Sensors Actuators, A Phys., vol. 85, pp. 335-339 (2000).

14 G. Gesele, J. Linsmeier, V. Drach, J. Fricke, and R. Arens-Fischer, J. Phys. D. Appl. Phys., vol. 30, no. 21, p. 2911 (1997).

15 C. Populaire, B. Remaki, V. Lysenko, D. Barbier, H. Artmann, and T. Pannek, Appl. Phys. Lett., vol. 83, no. 7, p. 1370 (2003).

16 S. P. Duttagupta, X. L. Chen, S. A. Jenekhe, and P. M. Fauchet, Solid State Commun., vol. 101, no. 1, pp. 33-37 (1997).

17 D. Bellet, P. Lamagnere, A. Vincent and Y. Brechet, J. Appl. Phys., vol. 80, $\mathrm{n}^{\circ}$ 7, p. 3772-3776 (1996).

18 S. Fakiria, A. Montagneb, K. Rahmounc, A. Iostb and K. Ziouchea, Mat. Sci. \& Eng. A 711, 470-475 (2018). 
19P. J. Newby, B. Canut, J.-M. Bluet, S. Gomès, M. Isaiev, R. Burbelo, K. Termentzidis, P. Chantrenne, L. G. Fréchette, and V. Lysenko, J. Appl. Phys., vol. 114, no. 1, p. 014903 (2013).

20 M. Isaiev, P. J. Newby, B. Canut, A. Tytarenko, P. Lishchuk, D. Andrusenko, S. Gomès, J.-M. Bluet, L. G. Fréchette, V. Lysenko, and R. Burbelo, Mater. Lett., vol. 128, pp. 71-74 (2014).

21 A. Cruz-Orea, I. Delgadillo, H. Vargas, A. Gudino-Martinez, E. Marin, C. VazquezLopez, A. Calderon and J.J. Alvarado-Gil, J. Appl. Phys. 79(12), 8951-8954 (1996).

$22 \mathrm{~S}$. Gomès, P. Newby, B. Canut, K. Termentzidis, O. Marty, L. Fréchette, P. Chantrenne, V. Aimez, J.M. Bluet, V. Lysenko, Microelectronics Journal 44(11), 1029-1034, (2013).

23 K. Valalaki and A.G. Nassiopoulou, J. Phys. D. : Appl. Phys, vol. 50, 195302 (2017)

24 J.H. Seol, D.S. Barth, J. Zhu, D. Coso, K. Hippalgaonkar, J. Lim, J. Han, X. Zhang, and A. Majumdar, Appl. Phys. Lett., vol. 111, 063104 (2017)

25 I. H. Campbell and P. M. Fauchet, Solid State Commun., vol. 58, no. 10, pp. 739741 (1986).

26 M. N. Islam, A. Pradhan, and S. Kumar, $J$. Appl. Phys., vol. 98, no. 2, p. 024309 (2005).

27 M. Massoud, B. Canut, P. Newby, L. Frechette, P.O. Chapuis, J.M. Bluet, Nuclear Instruments and Methods in Physics Research B, vol. 341, pp. 27-31 (2014).

28 B. Canut, M. Massoud, P. Newby, V. Lysenko, L. Frechette, J.M. Bluet, I. Monnet, Nuclear Instruments and Methods in Physics Research B, vol. 327, pp. 99102 (2014).

29 A. Meftah, F. Brisard, J. M. Costantini, E. Dooryhee, M. Hage-Ali, M. Hervieu, J. P. Stoquert, F. Studer, and M. Toulemonde, Phys. Rev. B, vol. 49, no. 18, pp. 1245712463 (1994).
$30 \mathrm{C}$. Trautmann, S. Klaumünzer, and $\mathrm{H}$. Trinkaus, Phys. Rev. Lett., vol. 85, no. 17, pp. 3648-3651 (2000).

31 D. Kanjilal, Current Science, vol. 80, No. 12, pp. 1560-1566 (2001).

32 Suzaki and A. Tachibana, Appl. Opt., vol. 14, pp. 2809-2810 (1975)

33 T. Hart, R. Aggarwal, and B. Lax, Phys. Rev. B, vol. 1, no. 2, pp. 638-642, (1970).

34 S. Huang, X.-D. Ruan, X. Fu and H.-Y. Yang, J Zhejiang Univ Sci A, 10(1), pp 716, (2009)

35 C. Populaire, "Propriétés physiques du silicium poreux: Traitements et applications aux microsystèmes," Ph.D. dissertation, INSA Lyon (2005).

36 G. Doerk, C. Carraro, and R. Maboudian, Phys. Rev. B, vol. 80, no. 7, pp. 1-4, 2009

$37 \mathrm{~J}$. von Behren and P. M. Fauchet, in Properties of Porous Silicon, edited by L. Canham (The IEE, London, 2006) 1st ed., Chap. 8.2, p. 405.

38 S. Gomes, A. Assy, P.-O. Chapuis, Physica Status Solidi (a), vol. 212, pp. 477-494 (2015)

39 M. Massoud, J. M. Bluet, V. Lacatena, M. Haras, J.-F. Robillard, and P.-O. Chapuis. Appl Phys. Lett., vol. 111, pp. 063106 (2017).

40 A.M. Massoud et al., Air contribution in calibrated microprobe-based scanning thermal microscopy, submitted

41 A. Delan, M. Rennau, S. E. Schulz, and T. Gessner, Microelectron. Eng., vol. 70, no. 2-4, pp. 280-284 (2003).

42 M. G. Burzo, P. L. Komarov, and P. E. Raad, IEEE Trans. Components Packag. Technol., vol. 26, no. 1, pp. 80-88 (2003). 43 M. B. Kleiner, S. A. Kuhn, and W. Weber, IEEE Trans. Electron Devices, vol. 43, no. 9, pp. 1602-1609 (1996).

44 Y. S. Touloukian, R. W. Powell, C. Y. Ho, and P. G. Klemens, Thermophysical Properties of Matter-The TPRC Data, Volume 2 (1971). 
45 Y. He, D. Donadio, and G. Galli, Appl. Phys. Lett., vol. 98, no. 14, p. 144101 (2011).

46 D. Li, Y. Wu, P. Kim, L. Shi, P. Yang, and A. Majumdar, Appl. Phys. Lett., vol. 83, no. 14, pp. 2934-2936 (2003).

47 A. Minnich and G. Chen, Appl. Phys. Lett., vol. 91, 073105 (2007).

48 R. Yang, G. Chen, M. Dresselhaus, Phys. Rev. B, vol. 72, pp. 125418 (2005)

49Z. Tian, K. Esfarjani, J. Shiomi, A.S. Henry, and G. Chen, Appl. Phys. Lett., vol. 99, pp. 053122 (2011).

50 L.B. Freund and S. Suresh, "Thin film materials", Cambridge University Press, 2003, p. 96. 


\section{Figures captions:}

FIG. 1. Raman spectrum for an IPSi sample ( $E_{1}$ energy and $3 \times 10^{13} \mathrm{~cm}^{-2}$ fluence). The red curve corresponds to the amorphous phase fit while the green one corresponds to the crystalline PSi.

FIG. 2. Scanning electron microscopy (a to f) images of (a,b,) non-IPSi, (c,d) IPSi with low fluence $10^{12}$ $\mathrm{cm}^{-2}$ and respectively $\mathrm{E}_{2}$ and $\mathrm{E}_{1}$ energies, (e,f) IPSi with high fluence $3 \times 10^{13} \mathrm{~cm}^{-2}$ and respectively $\mathrm{E}_{2}$ and $\mathrm{E}_{1}$ energies. High-resolution transmission electron microscopy $(\mathrm{g}, \mathrm{h})$ images of $(\mathrm{g})$ non-IPSi and (h) IPSi at $3 \times 10^{13} \mathrm{~cm}^{-2}$ fluence and $E_{1}$ energy.

FIG. 3. (a) $\mu$-RT principle. (b) Temperature field obtained by FEM on the top surface by accounting for absorption in the volume with a Gaussian beam. The inset provides simulated isothermal contours (four rectangles for mesh enhancement at the laser spot position are displayed).

FIG. 4. Temperature rise as a function of absorbed power from the incident laser beam in the case of non-IPSi (black squares) and of irradiated sample with fluence $3 \times 10^{13} \mathrm{~cm}^{-2}$ at $E_{1}$ energy (red squares). The straight lines are linear fit to the data including the $(0,0)$ point (i.e. no shift for zero power deposition).

FIG. 5. Principle of the determination of thermal conductivity by SThM: (a) tip far from contact, (b) tip in contact. The inset is an image of the approximate temperature field in the tip and around obtained from FEM. Maximal temperature is at the apex of the tip. (c) Variation of power dissipated in the probe between situations (a) and (b) as a function of thermal conductivity of reference materials, and determination of effective thermal conductivity of the sample.

FIG. 6. Effective thermal conductivity as a function of the irradiation fluence for PSi with $\mathrm{P}=$ $56 \%$ obtained by SThM and $\mu-\mathrm{RT}$. The violet color is for the SThM results, while the orange one is for the $\mu$-RT.

FIG. 7. Effective thermal conductivity as a function of the amorphous phase, and linear fits for the two energies and two experimental techniques. Green star and circle at 0 amorphous phase correspond to the non-IPSi sample. 
FIGURE 1

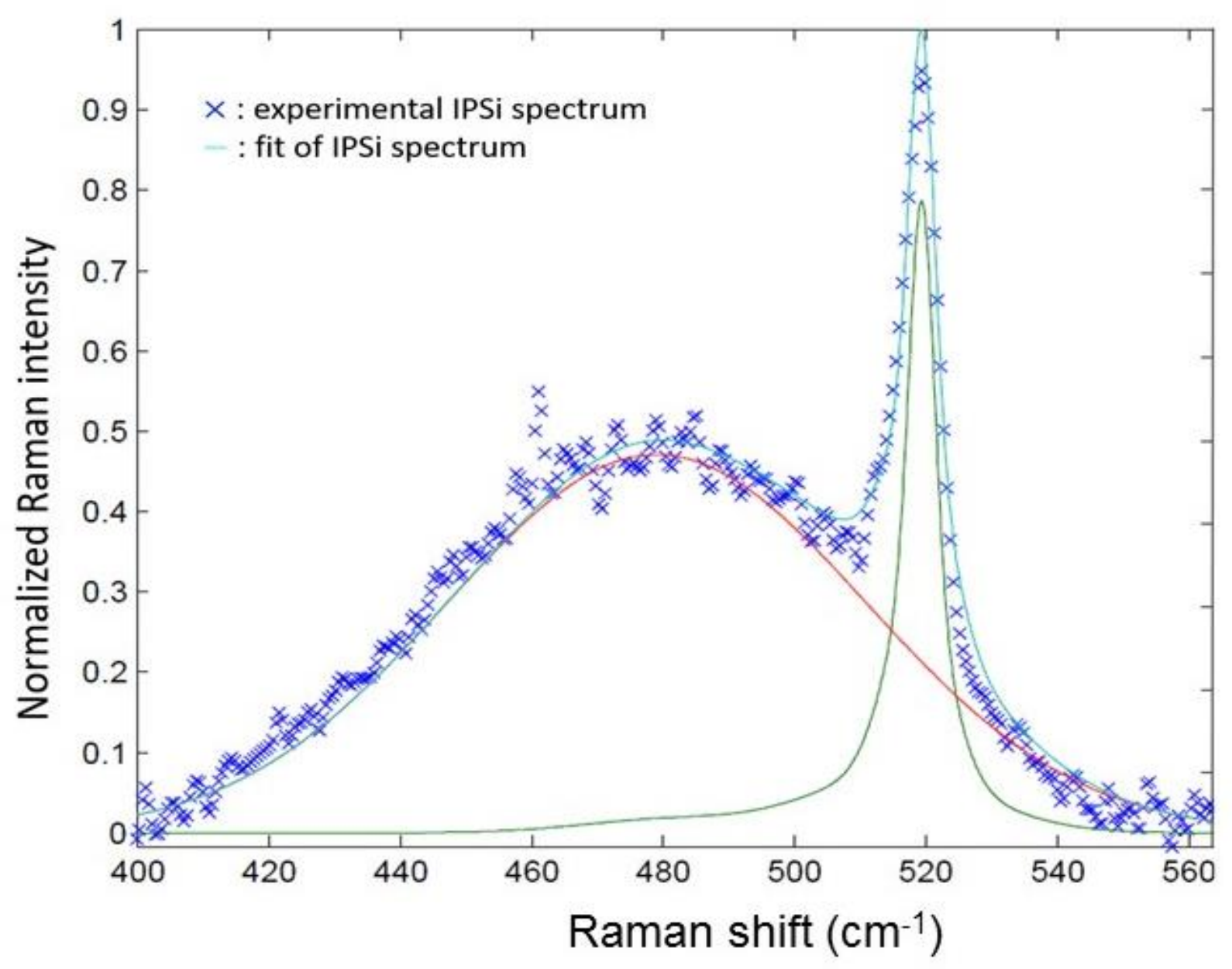


FIGURE 2
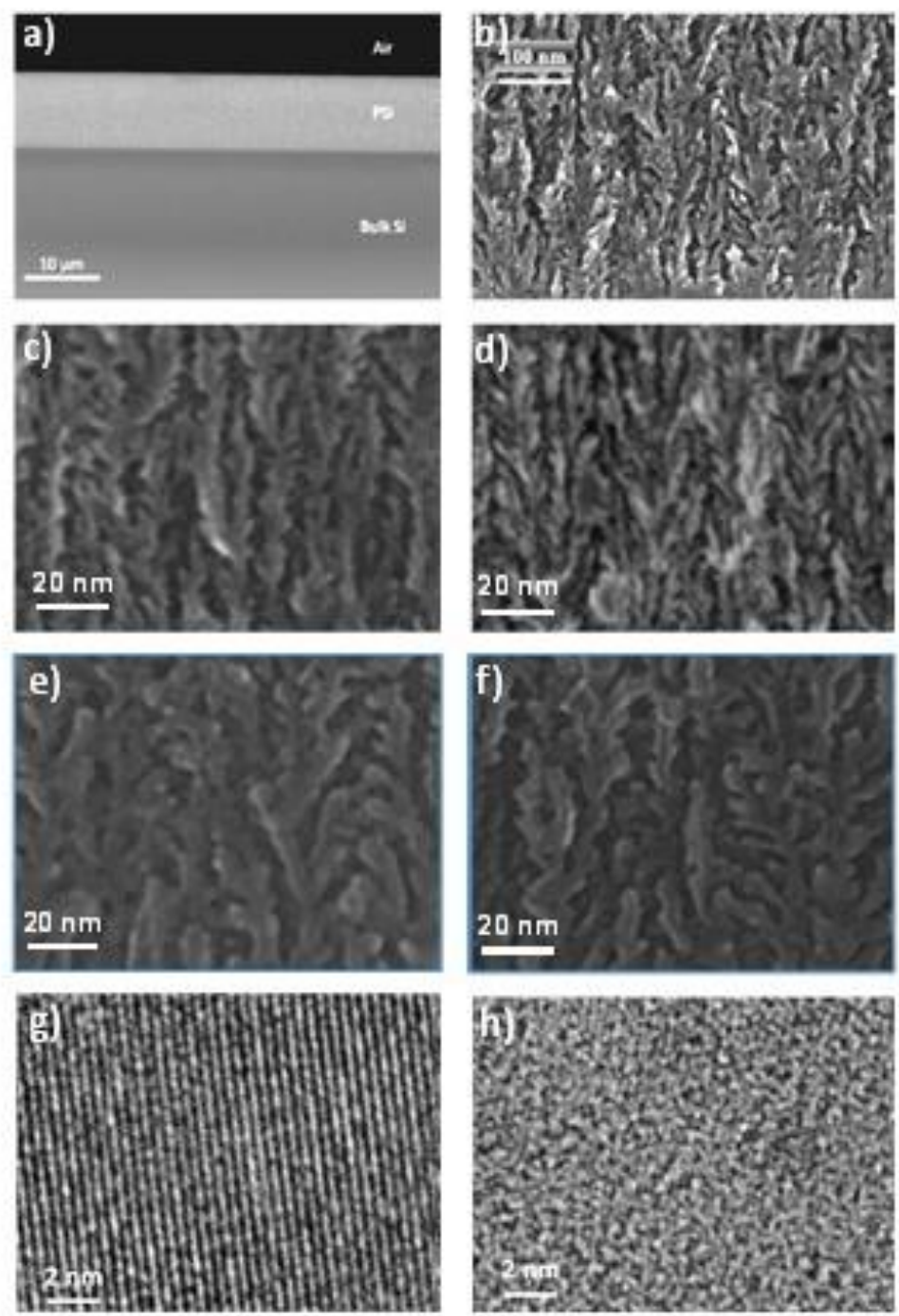


\section{FIGURE 3}

(a)

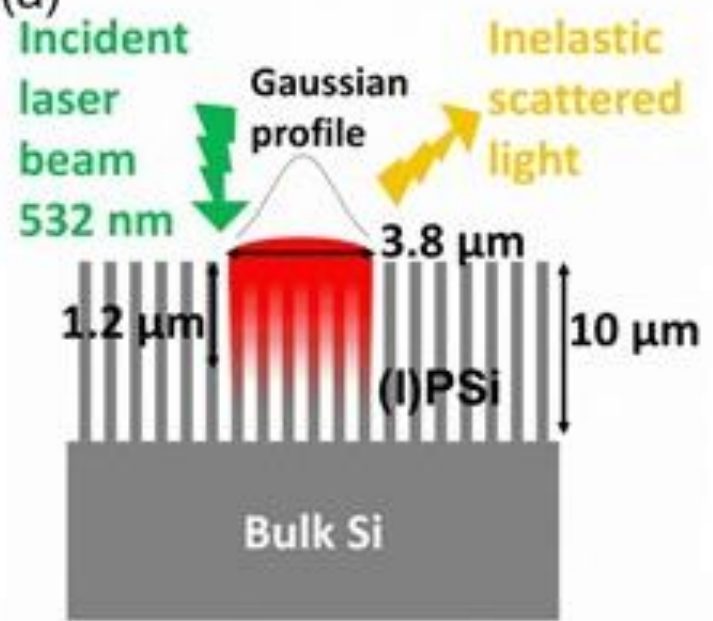

(b)

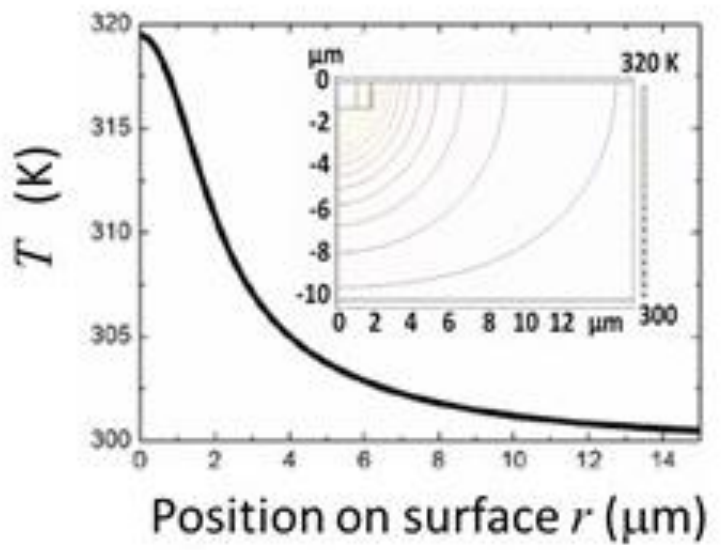




\section{FIGURE 4}

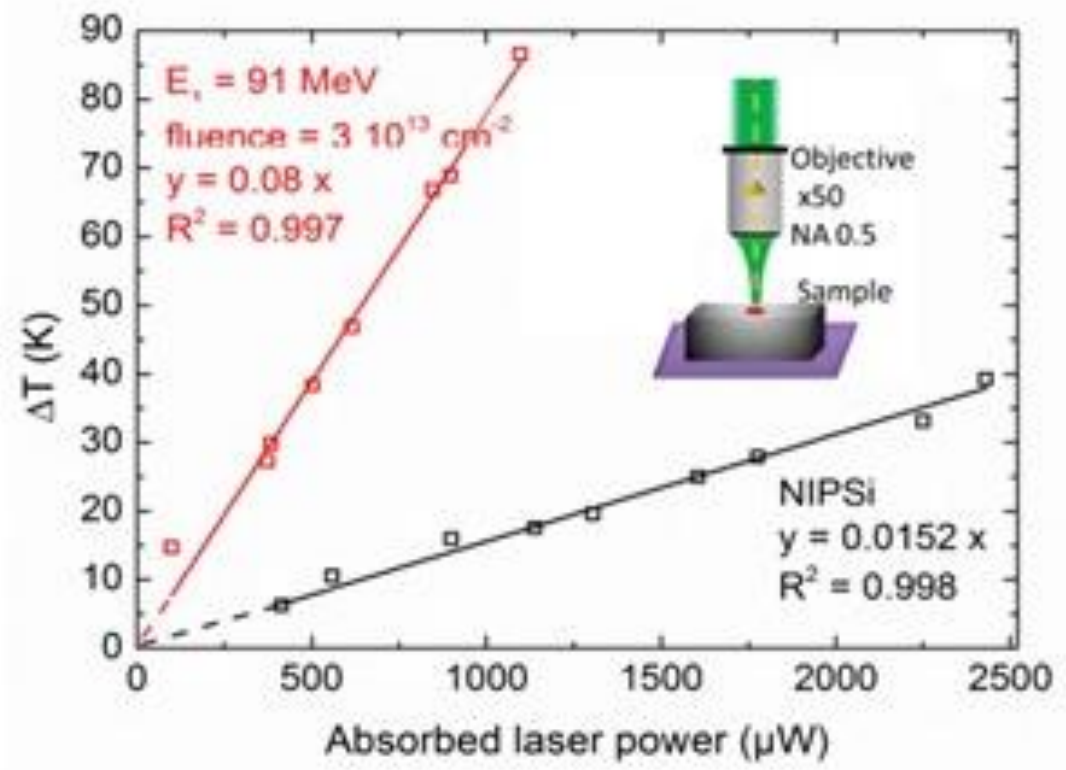


FIGURE 5
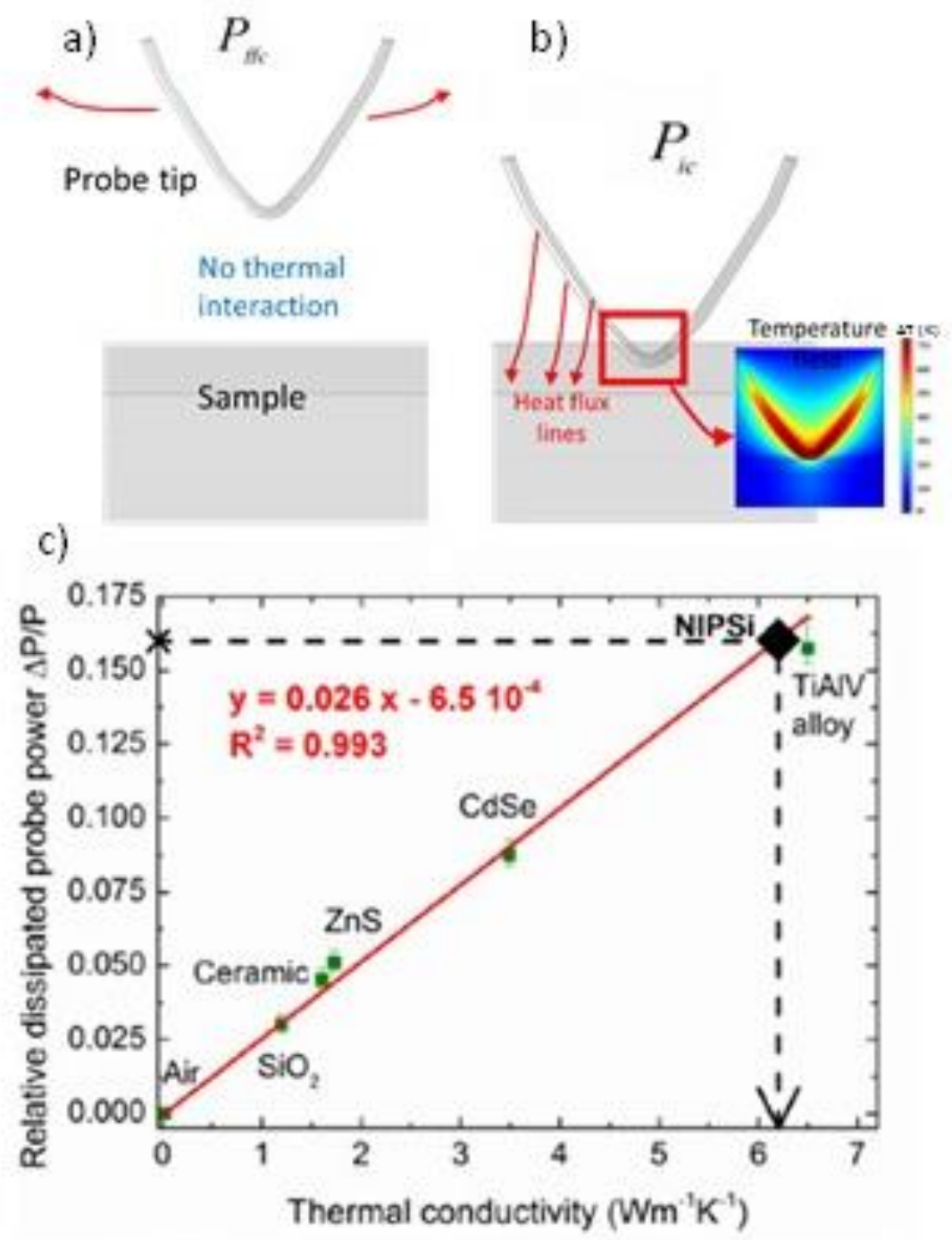
FIGURE 6

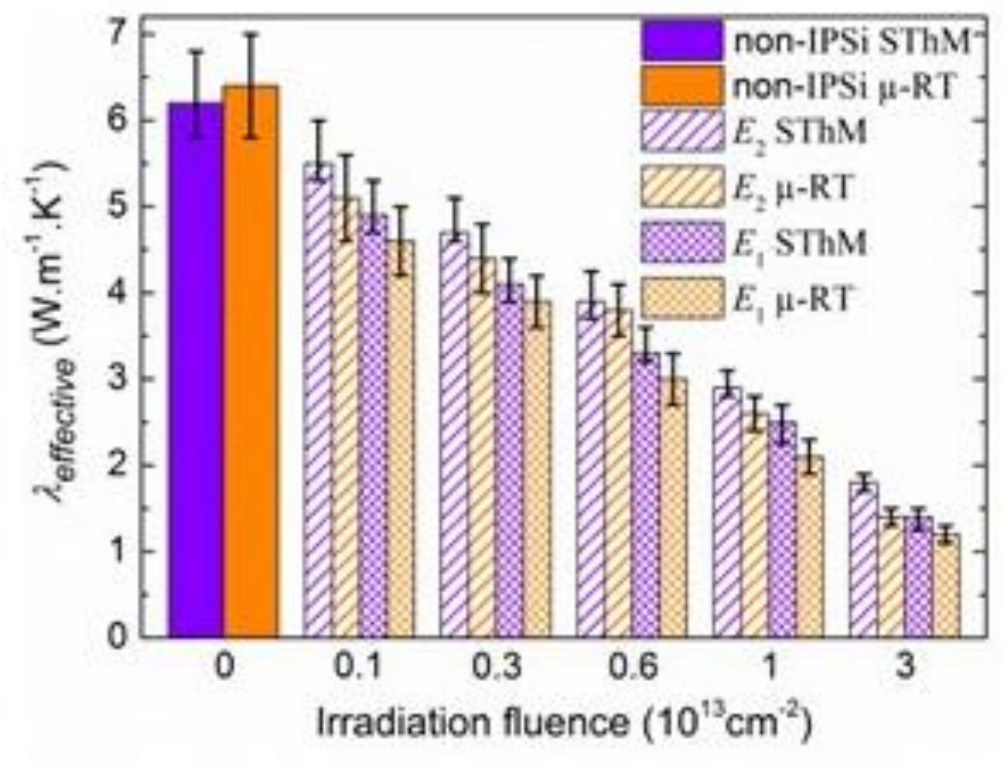


FIGURE 7

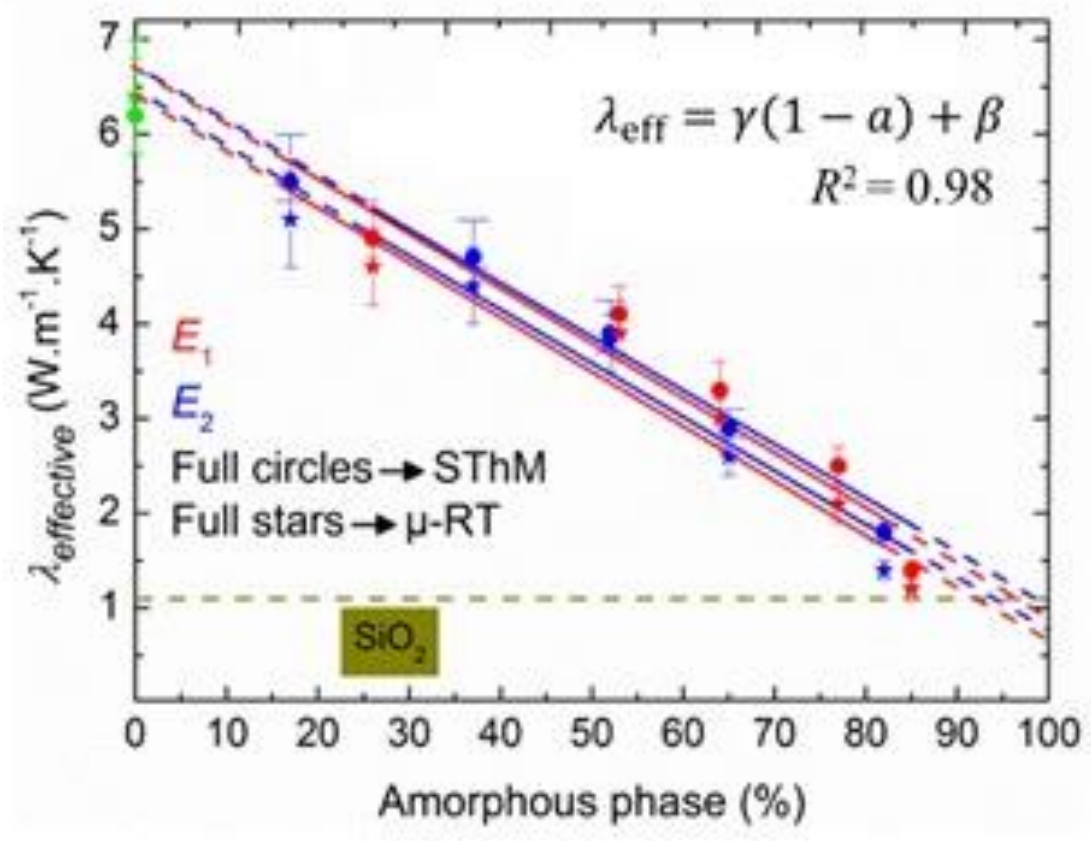

